УДК 621.396.677

\title{
МЕТОД РАСЧЁТА ШИРОКОПОЛОСНОЙ АНТЕННЫ БЕГУЩЕЙ ВОЛНЫ
}

\author{
Каращук Н.Н. \\ Военный институт им. С.П. Корольова, Житомир, Украина \\ Манойлов В.Ф., Морозов Д.С., Чухов В.В. \\ Житомирский государственный технологический университет, Житомир, Украина
}

Problems. Modern antennas range from 1 to $12 \mathrm{GHz}$ are often made in the form of waveguides or horns, which often makes the design of the antenna system too heavy and bulk. The possibility of creating broadband antennas in the printed version is described.

Objective. Vivaldi antenna as a broadband antenna in the printed version has been selected. But the construction of broadband electrodynamic model of Vivaldi slot antenna in its classical form is bulky and requires a new approach. A method of breaking a curved radiating slit into sections with a constant aperture width was described in this paper to solve the problem.

Methods. Solution of electrodynamic equations for the transverse components of the electric field strength for the regular sections of the antenna allows receiving the electrodynamic model of Vivaldi slot antenna.

Results. The results of calculations have been tested experimentally and are presented in the paper. A comparison of the calculated and experimental directional diagrams of slot broadband Vivaldi antenna at a frequency of $10 \mathrm{GHz}$ in two planes (E and $\mathrm{H}$ ) is shown. The corresponding directional diagrams have the difference of not more than $5-10 \%$, which confirms the effectiveness of the method.

Conclusions. The calculation method can be applied to a wide kind of curved planar antennas, and antenna as an effective replacement of bulky and heavy horn radiators.

Keywords: slot antenna; a planar antenna; antenna Vivaldi; methods of calculation.

\section{Введение}

В современной аппаратуре радио-мониторинга, работающего в диапазоне от 1 до 12 ГГц широко используются рупорные и антенны волноводного типа, являющиеся классическими антенными устройствами. Вместе с тем, на смену рупорным антеннам всё чаще приходят щелевые антенны в печатном исполнении, которые позволяют существенно снизить массогабаритные показатели антенны. При своих очевидных преимуществах широкополосные антенны требуют относительно громоздких вычислений $[1,2]$.

Целью данной работы является изложение более компактного метода определения параметров щелевой антенны Вивальди.

\section{Конструкция щелевой антенны}

В качестве замены громоздким рупорным антеннам предложена широкополосная щелевая антенна бегущей волны в печатном исполнении (рис.1)

Антенна выполнена на подложке $\left(\varepsilon_{r}=3,5\right)$ толщиной 0,25 мм и медной фольги, толщиной 0,035 мм. Раскрыв щели в самой широкой части равен 180 мм, а в месте запитки 0,5мм, длинна щели - 500 мм.
Антенное устройство запитывается в начале цепи посредством симметрирующего трансформатора, представляющего собой несимметричную нерегулярную полосковою линию, к несимметричному выходу которой присоединён коаксиальный разъём.

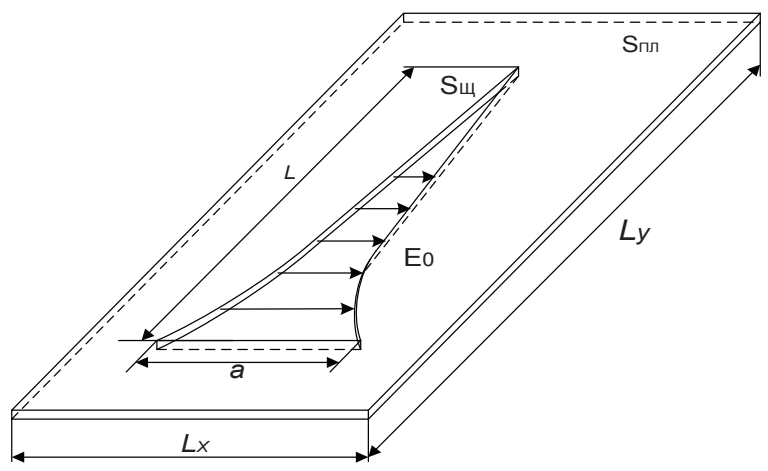

Рис.1. Широкополосная щелевая антенна бегущей волны в печатном исполнении

Построение электродинамической модели такой щелевой антенны довольно сложно. Однако, если представить, что в направляющей структуре распространяется только один тип волны (Е или Н), и что он не изменяется в следствии изменения поперечного сечения щели, то для анализа 
излучения такой антенны можно использовать подход, основанный на разбивании щели на участки с постоянной шириной раскрыва [3].

Представим расширяющую щелевую антенну (антенна Вивальди) в виде отдельных участков регулярных щелевых линий с постоянной шириной раскрыва. Используя подход из работы [4] для аппроксимации нерегулярной структуры предположим, что шаг увеличения ширины щели много меньше четверти длинны волны $\lambda_{0}$ (рис.2) :

$$
\omega_{n+1}-\omega_{n}=\Delta \omega<<\lambda_{0} / 4
$$

где $\omega_{\mathrm{n}}$ - ширина щели п-го участка направляющей структуры антенны, $\lambda_{0}$ - длина волны электромагнитных колебаний на входе антенны. Отдельные участки, на которые разбивалась щелевая антенна были длинной $\lambda_{0} / 8$.

Связь мощности $P$ переносимого в щели излучения с электромагнитным полем $\mathrm{E}_{\mathrm{X}}(0)$ в линии вдоль оси $x$ и волновым сопротивлением линии $\mathrm{Z}_{\mathrm{B}}$ на данной частоте определяется выражением

$$
\mathrm{P}=\left|E_{x}(0)\right|^{2} / Z_{B}
$$

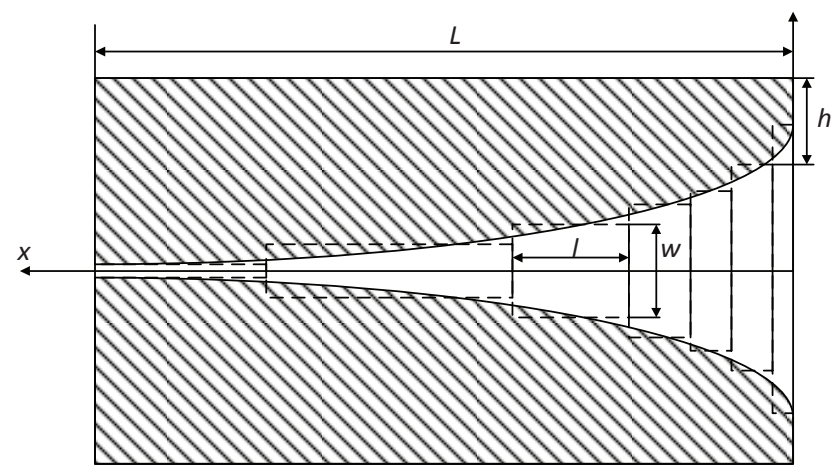

Рис.2. Аппроксимация расширяющейся направляющей структуры антенны регулярными участками

Для сохранения условия бегущей волны вдоль направляющей структуры антенны необходимо, чтобы выполнялось

$\sqrt{\mathrm{P}}=\left|a_{1}^{i-1}\right| / \sqrt{Z_{B}^{i-1}}=\left|a_{1}^{i}\right| / \sqrt{Z_{B}^{i}}=\left|a_{1}^{i+1}\right| / \sqrt{Z_{B}^{i+1}}=$ const $;$

где индексы означают номер участка нерегулярной линии.

Как показано в [4], поперечная компонента напряжённости электрического поля для $i$-го регулярного участка антенны (рис.2) определяется следующим выражением:

$$
\begin{gathered}
E_{x}^{(i)}\left(x^{\prime}, \mathrm{y}^{\prime}\right)=\mathrm{e}^{\left(j k_{x}^{(i)} z^{\prime}\right)}\left(\frac{2 \sqrt{Z_{B}^{(i)}}}{\pi \omega^{(i)}}\right) \times \\
\times \sum_{n=1}^{M_{x}^{(i)}} \frac{a_{n}^{(i)} T_{2(n-1)\left(\frac{2 x^{\prime}}{\omega^{(i)}}\right)}}{\sqrt{1-\left(\frac{2 x^{\prime}}{\omega^{(i)}}\right)^{2}}} e^{\left(j k_{x}^{(i)} z^{\prime}\right)} E_{a}^{(i)}\left(x^{\prime}\right)
\end{gathered}
$$

где

$$
E_{a}^{(i)}\left(x^{\prime}\right)=\left(\frac{2 \sqrt{Z_{B}^{(i)}}}{\pi \omega^{(i)}}\right) \sum_{n=1}^{M_{x}^{(i)}} \frac{a_{n}^{(i)} T_{2(n-1)}\left(\frac{2 x^{\prime}}{\omega^{(i)}}\right)}{\sqrt{1-\left(\frac{2 x^{\prime}}{\omega^{(i)}}\right)^{2}}}
$$

здесь $x^{\prime}, \mathrm{y}^{\prime}$-текущие координаты, $\omega^{(i)}$-ширина щели $i$-го участка антенны, $k_{x}^{(i)}$ - компонента волнового вектора вдоль оси $x$ на $i$-ом участке, $a_{n}^{(i)}$ - амплитуды базисных функций для $i$-го участка антенны, $T_{n}$ - полином Чебышева порядка $n, M_{x}^{(i)}$ - количество гармоник поля для $i$-го регулярного участка антенны.

Для расчёта поля по формуле (3) необходимо выразить все коэффициенты $a_{n}^{(i)}$ через $a_{1}^{(i)}$. При этом полагаем, что $a_{1}^{(i)}=1$, т.к. в нашем случае имеет место задача на поиск собственных значений, а не задача о возбуждении.

Результирующее полу в дальней зоне будет определяться суммарным вкладом каждого регулярного участка:

$$
E(\theta, \varphi)=\sum_{n=1}^{N} E_{n}(\theta, \varphi),
$$

где $E_{n}(\theta, \varphi)$ - вклад $n$-го регулярного участка направляющей структуры антенны в поле дальней зоны.

Расчётные формулы для нахождения напряжённости электрической составляющей электромагнитного поля в данном случае будут иметь вид

$$
\begin{gathered}
E_{\theta}^{(i)}(\theta)=E_{a}^{(i)}\left(k_{0} \omega^{(i)} \cos \theta\right) \sqrt{\frac{2}{\sin \theta}} \times \\
\times\left[e^{\left(-j k_{0} L c^{(i)}\right)}\left\{\frac{F^{*}\left(u_{h}^{(i)}\right)-F^{*}\left(u_{1}^{(i)}\right)}{\sqrt{c^{(i)}-\sin \theta}}\right\}+\right.
\end{gathered}
$$




$$
\left.+\Gamma e^{\left(-j k_{0} L c^{(i)}\right)}\left\{\frac{F^{*}\left(\bar{u}_{h}^{(i)}\right)-F^{*}\left(\bar{u}_{1}^{(i)}\right)}{\sqrt{c^{(i)}+\sin \theta}}\right\}\right]
$$

в Е-плоскости, и

$$
\begin{aligned}
& E_{\theta}^{(i)}(\varphi)=E_{a}^{(i)}(0)\left[\frac{\exp \left(-j k_{0} L c^{(i)}\right)}{c^{(i)}+\cos \varphi} \times\right. \\
& \times\left\{\sin \varphi\left[F\left(p_{h}^{(i)}\right) \exp \left(j v_{h}^{(i)}\right)-F\left(p_{1}^{(i)}\right) \exp \left(j v_{1}^{(i)}\right)\right]+\right. \\
& \left.+\sin (\varphi / 2) \sqrt{2\left(c^{(i)}-1\right)}\left[F\left(q_{h}^{(i)}\right)-F\left(q_{1}^{(i)}\right)\right]\right\}-\Gamma \frac{\exp \left(j k_{0} \mathrm{Lc}^{(i)}\right)}{c^{(i)}-\cos \varphi} \mathrm{Y} \\
& \mathrm{u}\left\{\sin \varphi\left[F\left(p_{h}^{(i)}\right) \exp \left(j \bar{v}_{h}^{(i)}\right)-F\left(p_{1}^{(i)}\right) \exp \left(j \bar{v}_{1}^{(i)}\right)\right]-\right. \\
& \left.\left.-\sin \left(\frac{\varphi}{2}\right) \sqrt{2\left(\mathrm{c}^{(\mathrm{i})}+1\right)}\left[\mathrm{F}\left(\overline{\mathrm{q}}_{\mathrm{h}}^{(\mathrm{i})}\right)-\mathrm{F}\left(\overline{\mathrm{q}}_{1}^{(\mathrm{i})}\right)\right]\right\}\right]
\end{aligned}
$$

в Н-плоскости, где Г-гамма-функция, $x_{1}^{(i)}$ и $x_{h}^{(i)}$ - координаты нижней и верхней границ $i$-го участка, $F(v)$ - интеграл Френеля, определяемый соотношением

$$
F(v)=\int_{0}^{v} \frac{\exp (-j t)}{\sqrt{2 \pi t}} d t
$$

Первый множитель в (6), (7) определяется выражением

$$
E_{a}^{(i)}(v)=\sqrt{Z_{0}^{(i)}} J_{0}(v),
$$

где $\mathrm{J}_{0}(\mathrm{v})$ - функция Бесселя первого рода нулевого порядка. Остальные компоненты, входящие в (6), (7), определяются выражениями

$$
\begin{gathered}
c^{(i)}=\lambda_{0} / \lambda_{i}^{\prime}, \quad k_{0}=2 \pi / \lambda_{0} ; \\
u_{a}^{(i)}=k_{0} x_{a}^{(i)}\left(c^{(i)}-\sin \theta\right), \\
\bar{u}_{a}^{(i)}=k_{0} x_{a}^{(i)}\left(c^{(i)}+\sin \theta\right) ; \\
v_{a}^{(i)}=k_{0} x_{a}^{(i)}\left(c^{(i)}+\cos \theta\right), \quad \bar{v}_{a}^{(i)}=k_{0} x_{a}^{(i)}\left(c^{(i)}-\cos \theta\right) ; \\
q_{a}^{(i)}=k_{0} x_{a}^{(i)}\left(c^{(i)}-1\right), \quad \bar{q}_{a}^{(i)}=k_{0} x_{a}^{(i)}\left(c^{(i)}+1\right) ; \\
p_{a}^{(i)}=k_{0} x_{a}^{(i)}(1+\cos \varphi) .
\end{gathered}
$$

где $\lambda_{i}^{\prime}$ - длина волны в пределах регулярного участка антенны, индекса $а$ принимает значения $h$ либо 1.
Результаты экспериментов

По приведённым выше соотношениям построены диаграммы направленности для нашего случая на частоте 12 ГГц (рис.3 и рис.4) и коэффициента усиления (рис.5).

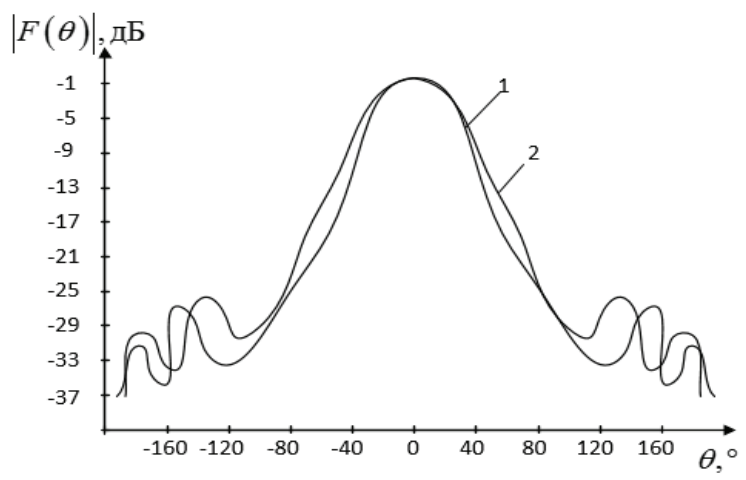

Рис.3 Расчётные (1) и экспериментальные (2) диаграммы направленности антенны на частоте 12 ГГц в Еплоскости

Видно, что в Е-плоскости уровень боковых лепестков не превышает -24 дБ, а в Н-плоскости -

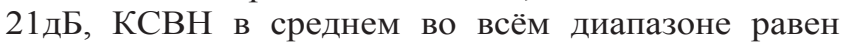
1,75 , коэффициент усиления 7-9дБ.Подобный характер диаграммы направленности сохраняется практически во всём указанном выше диапазоне частот.

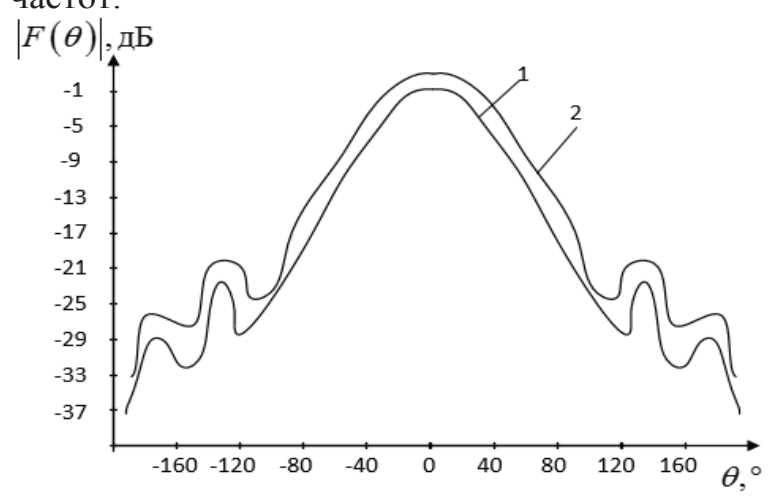

Рис.4 Расчётные (1) и экспериментальные (2) диаграммы направленности антенны на частоте 12 ГГц в Нплоскости

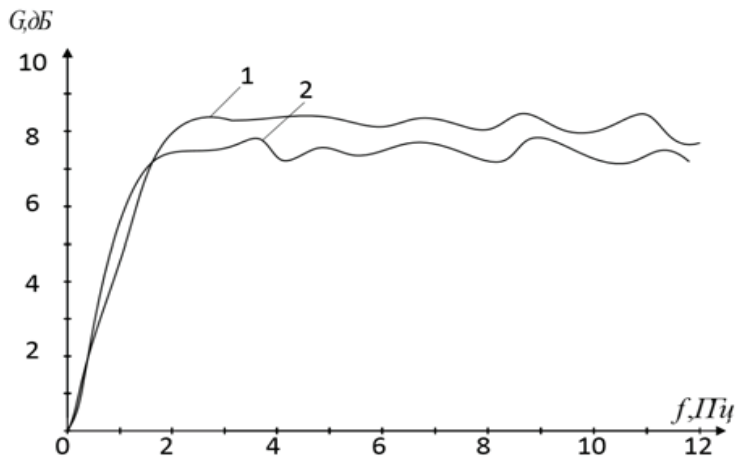

Рис.5 Расчётные (1) и экспериментальные (2) коэффициента усиления антенны в диапазоне до 12 ГГц 
Эксперименты показали, что когда волновые сопротивления в раскрыве антенны и у свободного пространства близки, отражение от концов щелевой части антенны всё же имеет место. Это происходит из-за скачка проводимости на границе антеннасвободное пространство. Поскольку на пути прохождения сигнала от области возбуждения до раскрыва антенны излучается лишь часть энергии сигнала в антенне, в результате отражения возникают колебания энергии в ней и формируются дополнительные колебания в принятом сигнале. В нашем случае мы использовали резистивные покрытия на проводящих пластинах антенны, обеспечивающих хорошее качество согласования. Расчёт таких покрытий является довольно сложной задачей, поэтому параметры покрытия подбирались экспериментально. Конечно, резистивные покрытия обеспечивают согласование, но их применение приводит к снижению КПД антенны.

КПД антенны определяется как отношение энергии, излучённой антенной $W_{\text {изл. }}$ к подводимой энергии $W_{\text {подв. }}$

$$
\text { КПД }=\frac{W_{\text {изл. }}}{W_{\text {подв. }}}
$$

В ходе исследований качество согласования антенны с фидером и свободным пространством оценивалось по величине КСВН в полосе частот. В результате применения резистивного покрытия, согласование удалось получить во всей полосе частот.

\section{Заключение}

Сравнительный анализ полученных результатов модели и экспериментальных исследований рассмотренной антенны показывает, что соответствующие диаграммы направленности имеют расхождения не более 5-10\%. На основе щелевой структуры бегущей волны возможно создание плоской антенны, не уступающей по эффективности объёмному рупорному облучателю в диапазоне рабочих частот.

\section{Литература}

1. Виленский А.Р. Метод анализа пространственновременных характеристик ищзлучения печатных щелевых антенн бегущей волны // Наука и образование. МГТУ им. Н.Э. Баумана. Электрон. Журн. 2014.№ 5. С. 139-154. Режим доступа http://technomag.bmstu.ru/doc/710740.html

2. Виленский А.Р., Чернышев С.Л. Анализ дисперсионных характеристик погонных параметров элементарных отрезков сверхширокополосных балансных печатных щелевых антенн бегущей волны // Наука и образование. МГТУ им. Н.Э. Баумана. Электрон. Журн. 2013.№ 4. С. 201-216. Режим доступа : http://technomag.bmstu.ru/doc/548171.html

3. Ашихмин. А.В. Оптимизация конструкции диэлектрического обтекателя с целью повышения эффективности сверх-широкополосной антенны Вивальди. //А.В. Ашихмин, А.С. Самодуров. Сб. Антенны, вып.10 (101), - М.: 2005, с.61-64.

4. Фролов. А.А. Изучение электродинамических характеристик антенн и антенных систем СВЧ диапазона. // А.А. Фролов, С.В. Гирич, В.П. Заярный. Из. Вузов. Радиофизика, т. LII, №4, 2009, с.328-335

Поступила в редакцию 16.09 .2016 
Каращук Н.Н., Манойлов В.П., Морозов Д.С., Чухов В.В. Метод розрахунку широкосмугової антени біжучої хвилі

Проблематика. Сучасні антени діапазону від 1 до 12 ГГц часто виконуються у вигляді хвилеводів або рупорів, що часто робить конструкцію антеної системи занадто важкою і об'ємною. В роботі описується можливість створення широкосмугової антени в друкованому виконанні.

Мета досліджень. В якості широкосмугової антени в друкованому виконанні була обрана антена Вівальді. Однак побудова електродинамічної моделі широкосмугового щілинної антени Вівальді в класичному вигляді занадто об'ємне і вимагає нового підходу. Для вирішення цього завдання в роботі викладено метод розбиття криволінійної випромінюючої щілини на ділянки з постійною шириною розкриву.

Методика реалізації. Рішення електродинамічних рівнянь для поперечних компонент напруженості електричного поля для регулярних ділянок антени дозволяють отримати електродинамічну модель щілинної антенни Вівальді.

Результати досліджень. Результати розрахунків були перевірені експериментально i наводяться в роботі. Демонструється порівняння розрахункових і експериментальних діаграм спрямованості широкосмугової щілинної антени Вівальді на частоті 10 ГГц в двох площинах (Е і Н). Відповідні діаграми спрямованості мають розбіжність не більше 5-10\%, що підтверджує ефективність методу.

Висновки. Методика розрахунку може бути застосовна для багатьох різновидів криволінійних планарних антен, а сама антена як ефективна заміна громіздким і важким рупорним випромінювачам.

Ключові слова: щілинна антена; планарная антена; антена Вівальді; методика розрахунку

Каращук Н.Н., Манойлов В.Ф., Морозов Д.С., Чухов В.В.

Метод расчёта широкополосной антенны бегущей волны

Проблематика. Современные антенны диапазона от 1 до 12 ГГц зачастую выполняются в виде волноводов или рупоров, что часто делает конструкцию антенной системы слишком тяжёлой и объёмной. Описывается возможность создания широкополосной антенны в печатном исполнении.

Цель исследований. В качестве широкополосной антенны в печатном исполнении была выбрана антенна Вивальди. Но построение электродинамической модели широкополосной щелевой антенны Вивальди в классическом виде громоздко и требует нового подхода. Для решения этой задачи в работе изложен метод разбития криволинейной излучающей щели на участки с постоянной шириной раскрыва.

Методика реализации. Решение электродинамических уравнений для поперечных компонент напряжённости электрического поля для регулярных участков антенны позволяют получить электродинамическую модель щелевой антенный Вивальди.

Результаты исследований. Результаты расчётов были проверены экспериментально и приводятся в работе. Демонстрируется сравнение расчётных и экспериментальных диаграмм направленности широкополосной щелевой антенны Вивальди на частоте 10 ГГц в двух плоскостях (Е и Н). Соответствующие диаграммы направленности имеют расхождение не более 5-10\%, что подтверждает эффективность метода.

Выводы. Методика расчёта может быть применима для широкого вида криволинейных планарных антенн, а антенна как эффективная замена громоздким и тяжёлым рупорным излучателям.

Ключевые слова: щелевая антенна; планарная антенна; антенна Вивальди; методика расчёта 